Pacific

Journal of

Mathematics

INTERSECTION OF CONJUGACY CLASSES WITH BRUHAT CELLS IN CHEVALLEY GROUPS

ERIch W. Ellers and Nikolai Gordeev

Volume $214 \quad$ No. 2

April 2004 


\title{
INTERSECTION OF CONJUGACY CLASSES WITH BRUHAT CELLS IN CHEVALLEY GROUPS
}

\author{
Erich W. Ellers and Nikolai Gordeev
}

\begin{abstract}
Let $G=\widetilde{G}(K)$ where $\widetilde{G}$ is a simple and simply-connected algebraic group that is defined and quasi-split over a field $K$. We investigate properties of intersections of Bruhat cells $B \dot{w} B$ of $G$ with conjugacy classes $C$ of $G$, in particular, we consider the question, when is $B \dot{w} B \cap C \neq \emptyset$.
\end{abstract}

\section{Introduction.}

Let $(G, B, N, S)$ be a Tits system. Some aspects of intersections of conjugacy classes of $G$ with Bruhat cells $B \dot{w} B$ have been investigated by several authors (see e.g., [St1], $[\mathbf{K}],[\mathbf{V}]$ and $[\mathbf{V S}])$. Here $w \in W=N /(B \cap N)$ and $\dot{w} \in N$ is a preimage of $w$ with respect to the natural surjection $N \rightarrow W$. In particular, it is desirable to learn how a conjugacy class $C$ of $G$ is related to those conjugacy classes $C_{w}$ of $W$ for which $B \dot{w} B \cap C \neq \emptyset$, where $w \in C_{w}$.

Here we deal with the case where $G$ is a Chevalley group, i.e., $G$ is the group of points $\widetilde{G}(K)$ of a simple algebraic group $\widetilde{G}$ that is defined and quasi-split over a field $K$, thus $G$ is a proper or a twisted Chevalley group (see $[$ St2]). Therefore, one can define a Tits system $(G, B, N, S)$, where $S=\left\{w_{\alpha_{i}} \mid \alpha_{i} \in \Pi\right\}$ for a simple root system $\Pi$ corresponding to $G$ ([St2] and $[\mathbf{C 1}])$.

A crucial step to investigate intersections $B \dot{w} B \cap C$ was done by R. Steinberg $[\mathbf{S t 1}$ ] who constructed the cross-section of regular conjugacy classes in $B \dot{w}_{S} B$, where $w_{S}$ is a Coxeter element of $W$ with respect to the fixed set of generators $S$ of $W$, i.e., $w_{S}$ is a product of elements in $S$ in any order, where each $s \in S$ occurs exactly once. The next natural step is to consider intersections of regular classes with cells of the form $B \dot{w} \dot{w}_{S} \dot{w}^{-1} B$. Here we prove the following:

Theorem 1.1. Let $\widetilde{G}$ be a simple and simply-connected algebraic group that is defined and quasi-split over a field $K$ and let $G=\widetilde{G}(K)$. Further, let $C \subset G$ be a conjugacy class of $G$ such that

$$
B \dot{w}_{S} B \cap C \neq \emptyset,
$$

where $w_{S}$ is a Coxeter element of $W$ with respect to $S$. Then $C$ intersects all cells of the form $B \dot{w} \dot{w}_{S} \dot{w}^{-1} B$, where $w \in W$. 
Note that the condition $B \dot{w}_{S} B \cap C \neq \emptyset$ implies that every element of $C$ is regular in $\widetilde{G}$, except the case when $\widetilde{G}$ is not split and has the type $A_{2 l}$ ([St1, Remark 8.8]). Condition $(*)$ holds, for instance, for regular conjugacy classes of $G$ in the following cases (as shown in Section 4):

(a) $G=S L_{n}(K)$;

(b) $K=\bar{K}$ (where $\bar{K}$ is the algebraic closure of $K$ ).

In the cases (c) to (f) below, the field $K$ is supposed to be perfect:

(c) $\widetilde{G}$ is split over $K$ and $C=\widetilde{C} \cap G$ for a conjugacy class $\widetilde{C}$ of $\widetilde{G}$;

(d) $\operatorname{dim} K \leq 1$ and $C$ is a semisimple class (here $\operatorname{dim} K$ is the homological dimension of $K$ );

(e) $\widetilde{G}$ is split over $K, C \cap B \neq \emptyset$, and $C$ is a semisimple class;

(f) $C$ is a unipotent class, char $K$ is not a bad prime for $\widetilde{G}$, and if $\widetilde{G}$ is not split, then $\widetilde{G}$ is not of type $A_{2 l}$.

Theorem 1.1 implies:

Corollary 1.2. Let $\widetilde{G}$ be a simple and simply-connected algebraic group that is defined and quasi-split over a field $K$ and let $G=\widetilde{G}(K)$. Further, let $C \subset G$ be a regular conjugacy class of $G$. If one of Conditions (a) to $(f)$ holds, then $C$ intersects all Bruhat cells of the form $B \dot{w} \dot{w}_{S} \dot{w}^{-1} B$.

Remark. The statement of the Corollary in Case (a) follows from the existence of a normal rational form. Case (b) follows from a much more general fact: Every regular conjugacy class of a simple algebraic group (i.e., $G=\widetilde{G}(\bar{K})$ ) intersects all Bruhat cells (see Appendix). Also, in Case (f), if $K$ is a finite field, then a theorem of Kawanaka $[\mathbf{K}]$ shows that any regular unipotent conjugacy class intersects all Bruhat cells.

Now let $X \subset S, W_{X}=\langle X\rangle$. By $w_{X}$ we denote a product (in any order) of elements of $X$, where each $x \in X$ occurs exactly once, i.e., $w_{X}$ is a Coxeter element of $W_{X}$ with respect to $X$. It is natural to consider intersections $B \dot{w} \dot{w}_{X} \dot{w}^{-1} B \cap C$ next. In [GS] it has been shown that $B \dot{w}_{X} B \cap C \neq \emptyset$ for some $X \subset S$ if $C$ is a semisimple class and $K$ is a finite field. Here we prove:

Theorem 1.3. Let $\widetilde{G}$ be a simple and simply-connected algebraic group that is defined and quasi-split over a perfect field $K$ such that $\operatorname{dim} K \leq 1$, and let $G=\widetilde{G}(K)$. Further, let $C \subset G$ be a noncentral semisimple conjugacy class of $G$. Then $C$ intersects all Bruhat cells of the form Biw $\dot{w}_{X} \dot{w}^{-1} B$ for some $X \subset S, X \neq \emptyset$.

Remark. This theorem generalizes Proposition 6 from [GS].

We thank the referee for drawing our attention to a result of Geck and Pfeiffer (see Proposition 3.3) which allows us to extend our results to all Chevalley groups. 


\section{S-Coxeter elements in Coxeter groups.}

Let $W$ be a finite group of orthogonal transformations of a Euclidean space $V$ generated by reflections. Then $W$ is a Coxeter group. Let $S=\left\{s_{1}, \ldots, s_{r}\right\}$ be a Coxeter system of generators of $W$, i.e., $s_{i}^{2}=1$ for every $i=1, \ldots, r$ and $\left(s_{i} s_{j}\right)^{m_{i j}}=1$ is the system of basic relations for the group $W$ (see [Bou, IV, 1]). Then every element of the form $s_{\pi(1)} s_{\pi(2)} \ldots s_{\pi(r)}$, where $\pi \in S_{r}$, is called a Coxeter element of $W$. All Coxeter elements of $W$ constructed for all possible Coxeter systems of generators are conjugate in $W$ (see [Bou, V, 6, Proposition 1]), and if $V^{W}=\{0\}$, each Coxeter element acts on $V \backslash\{0\}$ without fixed points ([Bou, V, 6, 2]).

Definition 2.1. Let $X \subset S$ and let $W_{X}$ be the subgroup of $W$ generated by $X$. Every element of $W$ that is conjugate to a Coxeter element in $W_{X}$ will be called a generalized Coxeter element of $W$.

Definition 2.2. For a fixed system $S$ of generators the elements of the form $s_{\pi(1)} s_{\pi(2)} \ldots s_{\pi(r)}$, where $|S|=r$, will be called $S$-Coxeter elements. If $X \subset S$, then $X$-Coxeter elements in $W_{X}$ will be called generalized $S$-Coxeter elements of $W$.

Let $l_{S}(w)$ be the $S$-length of $w$, i.e., the length of $w$ with respect to $S$. Obviously, a Coxeter element $w \in W$ is $S$-Coxeter if and only if $l_{S}(w)=r$. Below, we shall work with a fixed system $S$ and we shall write $l(w)$ instead of $l_{S}(w)$. We shall use the well-known fact that $l_{X}(w)=l_{S}(w)$ for any $w \in W_{X}$.

Example 2.3. Let $W=S_{4}$ and $S=\{(12),(23),(34)\}$. Then we have six Coxeter elements (4-cycles) in $W$. Among them there are four $S$-Coxeter elements:

$$
(12)(23)(34),(34)(23)(12),(23)(12)(34), \quad(12)(34)(23),
$$

and two elements that are not $S$-Coxeter elements:

$$
(23)(12)(23)(34)(23),(23)(34)(23)(12)(23) \text {. }
$$

Lemma 2.4. Let $w_{1}, w_{2}$ be two $S$-Coxeter elements of $W$. Then there exists a sequence $\sigma_{1}, \sigma_{2}, \ldots, \sigma_{n} \in S$ (possibly $\sigma_{i}=\sigma_{j}$ for $\left.i \neq j\right)$ such that

$$
w_{2}=\sigma_{n} \sigma_{n-1} \ldots \sigma_{1} w_{1} \sigma_{1} \sigma_{2} \ldots \sigma_{n}
$$

and $l\left(\sigma_{i} \sigma_{i-1} \ldots \sigma_{1} w_{1} \sigma_{1} \sigma_{2} \ldots \sigma_{i-1} \sigma_{i}\right)=r$ for every $i=1, \ldots, n$.

Proof. See [C2, Section 10.3].

\section{A condition for the intersection of a conjugacy class with Bruhat cells and Gauss cells.}

We are going to use the concepts of $S$-ascent and $S$-descent and derive some of their properties. The notion of descent was introduced and considered in 
[GP] (without the name "descent") as a binary relation between elements of conjugacy classes of Coxeter groups. The notion of ascent is dual to that of descent.

Definition 3.1. Let $w_{1}, w_{2} \in W$. We say that there exists an $S$-ascent (resp. $S$-descent) from $w_{1}$ to $w_{2}$ if there is a sequence $\sigma_{1}, \ldots, \sigma_{n} \in S$ such that

$$
w_{2}=\sigma_{n} \sigma_{n-1} \ldots \sigma_{1} w_{1} \sigma_{1} \sigma_{2} \ldots \sigma_{n}
$$

and

$$
\begin{aligned}
& l\left(\sigma_{i} \sigma_{i-1} \ldots \sigma_{1} w_{1} \sigma_{1} \sigma_{2} \ldots \sigma_{i}\right) \\
& \geq(\text { resp. } \leq) l\left(\sigma_{i-1} \ldots \sigma_{1} w_{1} \sigma_{1} \sigma_{2} \ldots \sigma_{i-1}\right)
\end{aligned}
$$

for every $i=1, \ldots, n$.

Remark. As before, we fix a set $S$ of generators for $W$. In $[\mathbf{G P}]$ an $S$-descent from an element $w \in W$ to an element $w^{\prime} \in W$ is denoted by $w \longrightarrow w^{\prime}$. It is logical to denote an $S$-ascent from $w^{\prime} \in W$ to $w \in W$ by $w \longleftarrow w^{\prime}$.

Definition 3.2. Let $C \subset W$ be a conjugacy class. We define

$$
l(C)=\min \{l(w) \mid w \in C\} .
$$

The following proposition is due to M. Geck and G. Pfeifer ([GP, Theorem 3.2.9.(a)]):

Proposition 3.3. Let $C \subset W=W(R)$ be a conjugacy class. Then for every $w \in C$ there exists an $S$-descent to an element $w^{\prime} \in C$ such that $l\left(w^{\prime}\right)=l(C)$.

Let $G$ be a Chevalley group (proper or twisted) corresponding to a root system $R$ in the sense of [St2]. We fix a simple root system $\Pi=\left\{\alpha_{1}, \ldots, \alpha_{r}\right\}$ and a corresponding Borel subgroup $B=H U$. Let $W=W(R)$ be the Weyl group of $G$ and $S=\left\{w_{\alpha_{1}}, \ldots, w_{\alpha_{r}}\right\}$ the corresponding Coxeter system of generators. By $X_{\alpha}$ we denote below a root subgroup of $G$ (see [St2]).

The meaning of Definition 3.1 becomes clear from the following:

Proposition 3.4. Let $g \in B \dot{w} B\left(r e s p . g \in B^{-} \dot{w} B\right)$ and let $w^{\prime} \in W$ be an element that is conjugate to $w$. If there exists an $S$-ascent (resp. $S$-descent) from $w$ to $w^{\prime}$, then there exists an element $g^{\prime} \in B \dot{w}^{\prime} B\left(\right.$ resp. $\left.B^{-} \dot{w}^{\prime} B\right)$ that is conjugate to $\mathrm{g}$.

Proof. We shall use the following lemma:

Lemma 3.5. Let $w \in W$. Suppose

$$
w\left(\alpha_{i}\right)<0 \text {, and } w^{-1}\left(\alpha_{i}\right)<0
$$

for some $\alpha_{i} \in \Pi$. Then either $w=w_{\alpha_{i}} w^{\prime} w_{\alpha_{i}}$, where $l\left(w^{\prime}\right)=l(w)-2$, or $w=w_{\alpha_{i}} w^{\prime}=w^{\prime} w_{\alpha_{i}}$, where $l\left(w^{\prime}\right)=l(w)-1$. 
Proof. The assumption $w^{-1}\left(\alpha_{i}\right)<0$ implies

$$
w=w_{\alpha_{i}} w_{1},
$$

where $l\left(w_{1}\right)=l(w)-1\left(\left[\mathbf{C 2}\right.\right.$, Section 2.2]). Suppose $w_{1}\left(\alpha_{i}\right)=\beta>0$. Since $w\left(\alpha_{i}\right)=w_{\alpha_{i}}(\beta)<0$, we have $\beta=\alpha_{i}$ and we have the second possibility. Now let $w_{1}\left(\alpha_{i}\right)<0$. Then $w_{1}=w^{\prime} w_{\alpha_{i}}$ where $l\left(w^{\prime}\right)=l\left(w_{1}\right)-1$ and we have the first possibility.

First, let $g \in B \dot{w} B$, then $g=b_{1} \dot{w} b_{2}$. We may assume $b_{1}=1$ and $b_{2}=u \in$ $U$. Also, it is sufficient to prove the assertion for an $S$-ascent of one step, i.e., $w^{\prime}=w_{\alpha} w w_{\alpha}$ for some $\alpha \in \Pi$. We can write $u=u_{\alpha} v$, where $u_{\alpha}$ is a root subgroup element corresponding to $\alpha$ and where $v \in U$ is an element that has no $\alpha$-factors in any decomposition into positive root subgroup elements.

If $u_{\alpha}=1$, then $u^{\prime}=\dot{w}_{\alpha} u \dot{w}_{\alpha}^{-1} \in U$ and

$$
g^{\prime}=\dot{w}_{\alpha} g \dot{w}_{\alpha}^{-1}=\left(\dot{w}_{\alpha} \dot{w} \dot{w}_{\alpha}^{-1}\right)\left(\dot{w}_{\alpha} u \dot{w}_{\alpha}^{-1}\right)=\dot{w}^{\prime} u^{\prime} \in B \dot{w}^{\prime} B .
$$

Let $u_{\alpha} \neq 1$. Suppose $\beta=w(\alpha)>0$. We may assume $\beta \neq \alpha$ (otherwise $\left.w^{\prime}=w_{\alpha} w w_{\alpha}^{-1}=w\right)$. We have $g=\dot{w} u_{\alpha} \dot{w}^{-1} \dot{w} v=u_{\beta} \dot{w} v$. Now we can consider the element $u_{\beta}^{-1} g u_{\beta}$ instead of $g$ which satisfies the previous condition $u_{\alpha}=$ 1.

Suppose $\beta=w(\alpha)<0$ and $\gamma=w^{-1}(\alpha)>0$. We have $g=\dot{w} u_{\alpha} v=$ $\dot{w} u_{\alpha} v u_{\alpha}^{-1} u_{\alpha}$. Note that $v^{\prime}=u_{\alpha} v u_{\alpha}^{-1}$ has no factors corresponding to $\alpha$. Consider now the element $\widetilde{g}=u_{\alpha} g u_{\alpha}^{-1}$ instead of $g$. We have $\widetilde{g}=u_{\alpha} \dot{w} v^{\prime}=$ $\dot{w} \dot{w}^{-1} u_{\alpha} \dot{w} v^{\prime}=\dot{w} u_{\gamma} v^{\prime}$, an element which also satisfies the condition $u_{\alpha}=1$.

Now let $\beta=w(\alpha)<0, \gamma=w^{-1}(\alpha)<0$. Then, by Lemma 3.5, either $w_{\alpha} w w_{\alpha}=w$ and, therefore, there is nothing to prove, or $l\left(w_{\alpha} w w_{\alpha}\right)<l(w)$ which contradicts our assumption.

Second, let $g \in B^{-} \dot{w} B$. We may assume $g=v v_{\alpha} \dot{w} u_{\alpha} u$, where $v \in U^{-}$, $v_{\alpha} \in X_{-\alpha}, u_{\alpha} \in X_{\alpha}, u \in U$ and the elements $v, u$ have no factors from the group $X_{ \pm \alpha}$. Note, $\dot{w}_{\alpha} v \dot{w}_{\alpha}^{-1} \in U^{-}, \dot{w}_{\alpha} u \dot{w}_{\alpha}^{-1} \in U$ (because $\alpha$ is a simple root). Thus, if $v_{\alpha}=u_{\alpha}=1$, then $\dot{w}_{\alpha} g \dot{w}_{\alpha}^{-1} \in B^{-} \dot{w}^{\prime} B$. Now put $\beta=w(\alpha), \gamma=w^{-1}(\alpha)$. If $\beta<0, \gamma<0$, we have $g=v v_{\alpha} \dot{w} u_{\alpha} \dot{w}^{-1} \dot{w} u=$ $v v_{\alpha} v_{\beta} \dot{w} u=v v_{\alpha} v_{\beta} v_{\alpha}^{-1} v_{\alpha} \dot{w} u=v\left(v_{\alpha} v_{\beta} v_{\alpha}^{-1}\right) \dot{w} u_{\gamma} u$, where $v_{\beta}=\dot{w} u_{\alpha} \dot{w}^{-1} \in X_{\beta}$, $u_{\gamma}=\dot{w}^{-1} v_{\alpha} \dot{w} \in X_{-\gamma}$. We may assume $\beta, \gamma \neq-\alpha$ (otherwise we have $\left.w_{\alpha} w w_{\alpha}=w\right)$. Thus the elements $v\left(v_{\alpha} v_{\beta} v_{\alpha}^{-1}\right), u_{\gamma} u$ have no factors from $X_{ \pm \alpha}$ and we are in the preceding case.

Let $\beta>0, \gamma<0$. Then $g=v v_{\alpha} u_{\beta} \dot{w} u=v_{\alpha} v^{\prime} \dot{w} u$, where the element $v^{\prime} \in$ $U^{-}$has no factor from $X_{-\alpha}$. Put $u_{\alpha}=\dot{w}_{\alpha} v_{\alpha} \dot{w}_{\alpha}^{-1}$. Then $\dot{w}_{\alpha} g \dot{w}_{\alpha}^{-1}=u_{\alpha} v^{\prime \prime} \dot{w}^{\prime} u^{\prime}$ for some $v^{\prime \prime} \in U^{-}, u^{\prime} \in U$. Thus $u_{\alpha}^{-1} \dot{w}_{\alpha} g \dot{w}_{\alpha}^{-1} u_{\alpha} \in B^{-} \dot{w}^{\prime} B$.

The case $\beta<0, \gamma>0$ is similar to the preceding one.

Let $\beta>0, \gamma>0$. Again, as above, we may assume $\beta, \gamma \neq \alpha$. Thus by Lemma 3.5, we have $l\left(w^{\prime}\right)=l\left(w_{\alpha} w w_{\alpha}\right)=l(w)+2$ which contradicts our assumption. 
Example 3.6. Let $G=S L_{3}(K)$ and let

$$
\dot{w}=\left(\begin{array}{rrr}
0 & 0 & 1 \\
0 & -1 & 0 \\
1 & 0 & 0
\end{array}\right), \quad \dot{w}^{\prime}=\left(\begin{array}{rrr}
0 & 1 & 0 \\
1 & 0 & 0 \\
0 & 0 & -1
\end{array}\right)
$$

Let $g$ be a semisimple element of $G$ that has no eigenvalues in $K$. Then $g$ is a regular element and therefore its conjugacy class $C_{g}$ intersects the big Bruhat cell $B \dot{w} B$ (see [EGH, Lemma 4]). But $C_{g} \cap B \dot{w}^{\prime} B=\emptyset$ because every element of the form $b_{1} \dot{w}^{\prime} b_{2}$ is conjugate to an element of the form

$$
\dot{w}^{\prime} b=\left(\begin{array}{rll}
0 & 1 & 0 \\
-1 & 0 & 0 \\
0 & 0 & 1
\end{array}\right)\left(\begin{array}{lll}
a_{11} & a_{12} & a_{13} \\
0 & a_{22} & a_{23} \\
0 & 0 & a_{33}
\end{array}\right)=\left(\begin{array}{rrr}
0 & a_{22} & a_{23} \\
-a_{11} & -a_{12} & -a_{13} \\
0 & 0 & a_{33}
\end{array}\right)
$$

which has an eigenvalue $a_{33} \in K$. Note that here $S=\left\{w_{12}, w_{23}\right\}$ (where $w_{i j}$ is the matrix in which the $i$ th and $j$ th elements of the standard basis are interchanged) and $l(w)=3, l\left(w^{\prime}\right)=l\left(w_{12}\right)=1$.

Example 3.7. Let $G=S L_{4}(K)$ and let

$$
\dot{w}=\left(\begin{array}{cccc}
0 & 1 & 0 & 0 \\
1 & 0 & 0 & 0 \\
0 & 0 & 0 & 1 \\
0 & 0 & 1 & 0
\end{array}\right), \quad \dot{w}^{\prime}=\left(\begin{array}{cccc}
0 & 0 & 0 & 1 \\
0 & 0 & 1 & 0 \\
0 & 1 & 0 & 0 \\
1 & 0 & 0 & 0
\end{array}\right) .
$$

Here $S=\left\{w_{12}, w_{23}, w_{34}\right\}$ and $\dot{w}=\dot{w}_{12} \dot{w}_{34}$ is a generalized $S$-Coxeter element. Thus every noncentral conjugacy class of $G$ intersects $B^{-} \dot{w} B([\mathbf{E G}])$. In particular, one can find a transvection $g \in B^{-} \dot{w} B$. But there are no transvections in $B^{-} \dot{w}^{\prime} B$ because $B^{-} \dot{w}^{\prime} B=\dot{w}^{\prime} B \dot{w}^{\prime}{ }^{-1} \dot{w}^{\prime} B=\dot{w}^{\prime} B$, and every matrix $x \in \dot{w}^{\prime} B$ satisfies the condition $\operatorname{rank}(x-1) \geq 2$.

The examples above show that if there is no $S$-ascent (resp. $S$-descent) from $w \in W$ to its conjugate $w^{\prime} \in W$, the condition $C \cap B \dot{w} B \neq \emptyset$ (resp. $C \cap$ $B^{-} \dot{w} B \neq \emptyset$ ) for a conjugacy class $C \in G$ does not necessarily imply $C \cap$ $B \dot{w}^{\prime} B \neq \emptyset$ (resp. $C \cap B^{-} \dot{w}^{\prime} B \neq \emptyset$ ).

Proposition 3.8. Let $g \in B \dot{w} B\left(\right.$ resp. $\left.g \in B^{-} \dot{w} B\right)$. Suppose $l\left(w_{\alpha} w w_{\alpha}\right)=$ $l(w)-2$ (resp. $\left.l\left(w_{\alpha} w w_{\alpha}\right)=l(w)+2\right)$. Then the conjugacy class $C_{g}$ of $g$ intersects either $B \dot{w}_{\alpha} \dot{w} \dot{w}_{\alpha}^{-1} B\left(\right.$ resp. $\left.B^{-} \dot{w}_{\alpha} \dot{w} \dot{w}_{\alpha}^{-1} B\right)$ or $B \dot{w}_{\alpha} \dot{w} B$ and $B \dot{w} \dot{w}_{\alpha} B$ (resp. $B^{-} \dot{w}_{\alpha} \dot{w} B$ and $\left.B^{-} \dot{w} \dot{w}_{\alpha} B\right)$.

Proof. Let $g \in B \dot{w} B$. We may assume, as in the proof of Proposition 3.4, that $g=\dot{w} u_{\alpha} u$ and $w=w_{\alpha} w_{1} w_{\alpha}$, where $l\left(w_{1}\right)=l(w)-2$. Moreover, $\beta=w_{1}(\alpha)>0, \gamma=w_{1}^{-1}(\alpha)>0$, and $w_{1}(\alpha), w_{1}^{-1}(\alpha) \neq \alpha$. If $u_{\alpha}=1$, then $\dot{w}_{\alpha} g \dot{w}_{\alpha}^{-1} \in B \dot{w}_{1} B$. Suppose $u_{\alpha} \neq 1$. Put $u_{-\alpha}=\dot{w}_{\alpha} u_{\alpha} \dot{w}_{\alpha}^{-1}$. There exists $u_{\alpha}^{\prime} \in X_{\alpha}$ (here $X_{\alpha}$ is the corresponding root subgroup) such that $u_{\alpha}^{\prime} u_{-\alpha}=\dot{w}_{\alpha} u_{\alpha}^{\prime \prime}$ for some $u_{\alpha}^{\prime \prime} \in X_{\alpha}$. Further, $g_{1}=\dot{w}_{\alpha} g \dot{w}_{\alpha}^{-1}=\dot{w}_{1} u_{-\alpha} u^{\prime}$ for some $u^{\prime} \in U$. Put $u_{\beta}=\dot{w}_{1} u_{\alpha}^{\prime} \dot{w}_{1}^{-1}$ (recall $\beta=w_{1}(\alpha)>0$ ). Then 
$g_{2}=u_{\beta} g_{1} u_{\beta}^{-1}=\dot{w}_{1} u_{\alpha}^{\prime} \dot{w}_{1}^{-1} \dot{w}_{1} u_{-\alpha} u^{\prime} u_{\beta}^{-1}=\dot{w}_{1} \dot{w}_{\alpha} u_{\alpha}^{\prime \prime} u^{\prime} u_{\beta}^{-1} \in B \dot{w}_{1} \dot{w}_{\alpha} B$. Since $l\left(w_{1} w_{\alpha}\right)=l\left(w_{\alpha} w_{1}\right)$, we also can find an element in $C_{g} \cap B \dot{w}_{\alpha} \dot{w}_{1} B$ (by Proposition 3.4).

Now let $g \in B^{-} \dot{w} B$. As in the proof of Proposition 3.4 we may assume $g=v v_{\alpha} \dot{w} u_{\alpha} u, \alpha \neq w(\alpha)>0, \alpha \neq w^{-1}(\alpha)>0$. If $v_{\alpha}=u_{\alpha}=1$, then $\dot{w}_{\alpha} g \dot{w}_{\alpha}^{-1} \in B^{-} \dot{w}_{\alpha} \dot{w} \dot{w}_{\alpha}^{-1} B$. Let $v_{\alpha}=1, u_{\alpha} \neq 1$. Then

$$
\begin{aligned}
g_{1}=\dot{w}_{\alpha} g \dot{w}_{\alpha}^{-1} & =\left(\dot{w}_{\alpha} v \dot{w}_{\alpha}^{-1}\right)\left(\dot{w}_{\alpha} \dot{w}_{\alpha}^{-1}\right)\left(\dot{w}_{\alpha} u_{\alpha} \dot{w}_{\alpha}^{-1}\right)\left(\dot{w}_{\alpha} u \dot{w}_{\alpha}^{-1}\right) \\
& =v^{\prime} \dot{w}_{\alpha} \dot{w} \dot{w}_{\alpha}^{-1} u_{-\alpha} u^{\prime},
\end{aligned}
$$

where $v^{\prime} \in U^{-}, u^{\prime} \in U, u_{-\alpha} \in X_{-\alpha}$. Moreover, the element $u^{\prime}$ has no factors in $X_{\alpha}$. Further, $u_{-\alpha} g_{1} u_{-\alpha}^{-1}=u_{-\alpha} v^{\prime} \dot{w}_{\alpha} \dot{w} \dot{w}_{\alpha}^{-1} u_{-\alpha} u^{\prime} u_{-\alpha}^{-1}$. Since $u_{-\alpha} u^{\prime} u_{-\alpha}^{-1} \in U$, we have $u_{-\alpha} g_{1} u_{-\alpha}^{-1} \in B^{-} \dot{w}_{\alpha} \dot{w} \dot{w}_{\alpha}^{-1} B$. Similar considerations work in the case $v_{\alpha} \neq 1, u_{\alpha}=1$.

Let $v_{\alpha} \neq 1, u_{\alpha} \neq 1$. Put $u_{\alpha}^{\prime}=\dot{w}_{\alpha} v_{\alpha} \dot{w}_{\alpha}^{-1}, v_{\alpha}^{\prime}=\dot{w}_{\alpha} u_{\alpha} \dot{w}_{\alpha}^{-1}, v^{\prime}=\dot{w}_{\alpha} v \dot{w}_{\alpha}^{-1}$, $u^{\prime}=\dot{w}_{\alpha} u \dot{w}_{\alpha}^{-1}$. Then

$$
g_{1}=\dot{w}_{\alpha} g \dot{w}_{\alpha}^{-1}=v^{\prime} u_{\alpha}^{\prime} \dot{w}_{\alpha} \dot{w} \dot{w}_{\alpha}^{-1} v_{\alpha}^{\prime} u^{\prime}=v^{\prime} u_{\alpha}^{\prime} \dot{w}_{\alpha} \dot{w} \dot{w}_{\alpha}^{-1}\left(v_{\alpha}^{\prime} u^{\prime} v_{\alpha^{\prime}}^{-1}\right) v_{\alpha}^{\prime} .
$$

Put $u^{\prime \prime}=v_{\alpha}^{\prime} u^{\prime} v_{\alpha^{\prime}}^{-1}, v^{\prime \prime}=v_{\alpha}^{\prime} v^{\prime}$. Then $g_{2}=v_{\alpha}^{\prime} g_{1} v_{\alpha^{\prime}}^{-1}=v^{\prime \prime} u_{\alpha}^{\prime} \dot{w}_{\alpha} \dot{w} \dot{w}_{\alpha}^{-1} u^{\prime \prime}$. Further, $u_{\alpha}^{\prime} \dot{w}_{\alpha}=x_{-\alpha} x_{\alpha}$ for some $x_{-\alpha} \in X_{-\alpha}, x_{\alpha} \in X_{\alpha}$. Hence

$$
g_{2}=v^{\prime \prime} x_{-\alpha} x_{\alpha} \dot{w} \dot{w}_{\alpha}^{-1} u^{\prime \prime}=v^{\prime \prime} x_{-\alpha} \dot{w} \dot{w}_{\alpha}^{-1}\left(\dot{w}_{\alpha} \dot{w}^{-1} x_{\alpha} \dot{w} \dot{w}_{\alpha}^{-1}\right) u^{\prime \prime} .
$$

Since $w^{-1}(\alpha)>0$ and $w^{-1}(\alpha) \neq \alpha$, we get $\dot{w}_{\alpha} \dot{w}^{-1} x_{\alpha} \dot{w} \dot{w}_{\alpha}^{-1} \in U$ and therefore $g_{2} \in B^{-} \dot{w} \dot{w}_{\alpha} B$. From Proposition 3.4 we get $C_{g} \cap B^{-} \dot{w}_{\alpha} \dot{w} B \neq \emptyset$.

\section{Proofs of the Theorems.}

Here $\widetilde{G}$ is a simple algebraic group defined and quasi-split over a field $K, \widetilde{B}=$ $\widetilde{T} \widetilde{U}$ is a Borel subgroup defined over $K, \widetilde{N}=N_{\widetilde{G}}(\widetilde{T}), \widetilde{W}=\widetilde{N} / \widetilde{T}$ and $G=$ $\widetilde{G}(K), B=\widetilde{B}(K), T=\widetilde{T}(K), U=\widetilde{U}(K), N=\widetilde{N}(K), W=N / T$. Further, let $\widetilde{\Pi}=\left\{\gamma_{1}, \ldots, \gamma_{s}\right\}$ be a simple root system of $\widetilde{G}$ and $\Pi=\left\{\alpha_{1}, \ldots, \alpha_{r}\right\}$ be a simple root system (in the sense of $[\mathbf{C 2}]$ ) for $G$ (which is obtained from $\widetilde{\Pi}$ by gluing of some roots).

Proof of Theorem 1.1. Assume that Condition (*) of Theorem 1.1 holds. Further let $C_{c} \subset W$ be the conjugacy class of Coxeter elements and let $\omega \in C_{c}$. By Proposition 3.3 there exists an $S$-descent from $\omega$ to an element $\omega^{\prime} \in C_{c}$ such that $l\left(\omega^{\prime}\right)=l\left(C_{c}\right)=r$. Since among the factors of $\omega^{\prime}$ there are all reflections $w_{\alpha_{i}}, \alpha_{i} \in \Pi$, the element $\omega^{\prime}$ is an $S$-Coxeter element. By Lemma 2.4 we have an $S$-ascent from $w_{S}$ (recall that $w_{S}$ is the Coxeter element from Condition $(*))$ to $\omega^{\prime}$ and, therefore, we have an $S$-ascent from $w_{S}$ to $\omega$. Now our statement follows from Proposition 3.4.

Condition $(*)$. Below, $K$ is a perfect field.

We need the following simple (and known) facts: 
Lemma 4.1. Let $\widetilde{C}$ be a conjugacy class of $\widetilde{G}$ such that $C=\widetilde{C} \cap G \neq \emptyset$. Further, let $g \in C$. If $H^{1}\left(K, C_{\widetilde{G}}(g)\right)=1$ then $C$ is a conjugacy class of $G$ (here $C_{\widetilde{G}}(g)$ is the centralizer of $g$ in $\widetilde{G}$ ).

Proof. The argument here is the same as in ([C2, Proposition 3.7.3]). Indeed, if $g^{\prime} \in C$, then there exists an element $\gamma \in \widetilde{G}$ such that $g^{\prime}=\gamma g \gamma^{-1}$. Thus, for every element $\sigma \in \operatorname{Gal}(\bar{K} / K)$ of the Galois group we have

$$
\sigma(\gamma) g \sigma\left(\gamma^{-1}\right)=\gamma g \gamma^{-1}
$$

and therefore $x_{\sigma}=\gamma^{-1} \sigma(\gamma) \in C_{\widetilde{G}}(g)$. Since $x_{\sigma}$ is a 1-cocycle, we have $x_{\sigma}=$ $y \sigma\left(y^{-1}\right)$ for some $y \in C_{\widetilde{G}}$ and therefore $\sigma(\gamma y)=\gamma y$ for every $\sigma \in \operatorname{Gal}(\bar{K} / K)$. Thus, $\gamma y \in G$ and $g^{\prime}=\gamma y g y^{-1} \gamma^{-1}$.

Lemma 4.2. Let $\widetilde{C}$ be a semisimple conjugacy class of $\widetilde{G}$ and let $C=$ $\widetilde{C} \cap G \neq \emptyset$. If $\operatorname{dim} K \leq 1$, then $C$ is a conjugacy class of $G$.

Proof. Since $\widetilde{G}$ is simply-connected, $C_{\widetilde{G}}(s)$ is a connected reductive group for $s \in \widetilde{C} \cap G\left([\mathbf{C 2}\right.$, Theorem 3.5.6] $)$ and therefore $H^{1}\left(K, C_{\widetilde{G}}(s)\right)=1([\mathbf{S t 1}$, 11.2]). Now the assertion follows from Lemma 4.1.

Lemma 4.3. Let $C$ be the same as in the preceding lemma. Suppose that $\widetilde{G}$ is split and $\widetilde{C}$ is a regular semisimple class such that $\widetilde{C} \cap T \neq \emptyset$. Then $C$ is a conjugacy class of $G$.

Proof. If $s \in \widetilde{C} \cap T$, then $C_{\widetilde{G}}(s)=\widetilde{T}$ is a $K$-split torus and therefore $H^{1}\left(K, C_{\widetilde{G}}(s)\right)=1$ ([Sp, 12.3.5.(3)]). Now the assertion follows from Lemma 4.1 .

Lemma 4.4. Let $u_{1}, u_{2} \in G$ be two regular unipotent elements of $\widetilde{G}$. Assume that char $K$ is not a bad prime for $\widetilde{G}$. Then there exist elements $t \in \widetilde{T}$ and $\gamma \in G$ such that $u_{1}=t \gamma u_{2} \gamma^{-1} t^{-1}$.

Proof. Let $\bar{G}=\widetilde{G} / Z(\widetilde{G}), \bar{T}=\widetilde{T} / Z(\widetilde{G})$. Then $\bar{G}$ is defined and quasi-split over $K$ and $Z(\bar{G})=1$. Further, let $u \in \widetilde{G}$ be a regular unipotent element and let $\bar{u}$ be its image in $\bar{G}$. The char $K$ is not a bad prime for $\bar{G}$, thus $V=C_{\bar{G}}(\bar{u})$ is a connected unipotent subgroup of $\bar{G}$ ([C2, Proposition 5.1.6]) which is defined and split over $K([\mathbf{S p}, 14.3 .8])$ and therefore $H^{1}(K, V)=$ 1 ([Sp, 12.3.5.(3)]). Hence any two regular unipotent elements of $\bar{G}(K)$ are conjugate (Lemma 4.1). If $\bar{G}_{1}(K) \leq \bar{G}(K)$ is a subgroup generated by unipotent elements of $\bar{G}$, then it is a normal subgroup and $\bar{G}(K)=$ $\bar{G}_{1}(K) \bar{T}(K)$ (this follows from the Bruhat decomposition). Now let $\bar{u}_{1}, \bar{u}_{2} \in$ $\bar{G}(K)$ be images of regular unipotent elements $u_{1}, u_{2} \in G$. Then there exist elements $\bar{\gamma} \in \bar{G}_{1}(K), \bar{t} \in \bar{T}(K)$ such that $\bar{u}_{1}=\bar{t} \bar{\gamma} \bar{u}_{2} \bar{\gamma}^{-1} \bar{t}^{-1}$. If $\gamma \in \widetilde{G}(K)=$ $G, t \in \widetilde{T}$ are preimages of $\bar{\gamma}, \bar{t}$, then $u_{1} \equiv t \gamma u_{2} \gamma^{-1} t^{-1}(\bmod Z(\widetilde{G}))$. Since $u_{1}, u_{2}$ are both unipotent elements, we have $u_{1}=t \gamma u_{2} \gamma^{-1} t^{-1}$. 
Now we check Condition $(*)$ for $($ a) to $(\mathrm{f})$ :

(a) If $G=S L_{n}(K)$, Condition $(*)$ is an immediate consequence of the representation of elements of $G L_{n}(K)$ in rational canonical form.

(b) Consider the case where $K$ is an algebraically closed field. According to Steinberg's theorem ([St1, 1.4]), the set

$$
\mathfrak{N}=\dot{w}_{\gamma_{1}} X_{\gamma_{1}} \dot{w}_{\gamma_{2}} X_{\gamma_{2}} \ldots \dot{w}_{\gamma_{s}} X_{\gamma_{s}}
$$

is a cross-section of all regular conjugacy classes of the group $\widetilde{G}$, where $\dot{w}_{\gamma_{1}}, \ldots, \dot{w}_{\gamma_{s}}$ is any fixed system of preimages of the basic reflections $w_{\gamma_{1}}, \ldots$, $w_{\gamma_{s}}$ in any fixed order (here $X_{\gamma_{i}}$ is the corresponding root subgroup). Moreover, we can rewrite $\mathfrak{N}$ in the form

$$
\mathfrak{N}=\dot{w}_{\gamma_{1}} \dot{w}_{\gamma_{2}} \ldots \dot{w}_{\gamma_{s}} X_{\theta_{1}} X_{\theta_{2}} \ldots X_{\theta_{s}}
$$

where $\theta_{i}=w_{\gamma_{s}} \ldots w_{\gamma_{i+2}} w_{\gamma_{i+1}}\left(\gamma_{i}\right)>0$. Since $K$ is an algebraically closed field, $\left\{\alpha_{1}, \ldots, \alpha_{r}\right\}=\left\{\gamma_{1}, \ldots, \gamma_{s}\right\}$ and any element in the intersection $C \cap$ $\tilde{N}(K)$ lies in the $S$-Coxeter cell $B \dot{w}_{\alpha_{1}} \dot{w}_{\alpha_{2}} \ldots \dot{w}_{\alpha_{r}} B$. This proves (*).

(c) If $\widetilde{G}$ is split over $K$, the closed subset $\mathfrak{N}$ (defined above) of $\widetilde{G}$ is defined over $K$ and $\mathfrak{N} \cap \widetilde{C} \in G$ ([St1, Section 9$])$.

(d) There exists a closed subset $\mathfrak{N}^{\prime}$ of $\mathfrak{N}$ which is defined over $K$ and such that every regular semisimple conjugacy class $\widetilde{C}$ of $\widetilde{G}$ intersects $\mathfrak{N}^{\prime}$ in just one point (and this point belongs to $G$ if $\widetilde{C} \cap G \neq \emptyset)([\mathbf{S t 1}, 9.11])$. Since $\mathfrak{N} \subset B \dot{w}_{S} B$ for some $S$-Coxeter element $w_{S}$, the assertion follows from Lemma 4.2.

(e) We may use the same argument as in (d), and Lemma 4.3.

(f) If $\widetilde{G}$ is split or $\widetilde{G}$ is not of type $A_{2 l}$, the cross-section of regular classes $\mathfrak{N}$ is defined over $K$ and for the conjugacy class of regular unipotent elements $\widetilde{C}$ we have $u=\widetilde{C} \cap \mathfrak{N} \in B \dot{w}_{S} B$, where $\dot{w}_{S} \in N$ for some $S$-Coxeter element $w_{S}$ in $W\left(\left[\mathbf{S t 1}\right.\right.$, Section 9]). Now let $u^{\prime} \in \widetilde{C} \cap G$. By Lemma 4.4 we have $t \gamma u^{\prime} \gamma^{-1} t^{-1}=u=u_{1} \dot{w}_{S} b_{1}$ for some $t \in \widetilde{T}, \gamma \in G$ and $u_{1} \in U, b_{1} \in B$. Hence $u^{\prime \prime}=\gamma u^{\prime} \gamma^{-1}=\left(t^{-1} u_{1} t\right)\left(t^{-1} \dot{w}_{S} t\right)\left(t^{-1} b_{1} t\right)$. Thus $u^{\prime \prime} \in \widetilde{B} \dot{w}_{S} \widetilde{B}$. But $u^{\prime \prime} \in G$ and, therefore, $u^{\prime \prime} \in B \dot{w} B$ for some $\dot{w} \in N$. Since $B \dot{w} B \subset \widetilde{B} \dot{w} \widetilde{B}$, we have $w=w_{S}$. This implies that the conjugacy class $C$ of $u^{\prime}$ in $G$ has a nontrivial intersection with $B \dot{w}_{S} B$, where $\dot{w}_{S} \in N$.

Proof of Theorem 1.3.

Below, $\widetilde{\Gamma}$ is a connected reductive algebraic group defined over a perfect field $K$ such that $\operatorname{dim} K \leq 1$.

Lemma 4.5. Let $\widetilde{P}=\widetilde{L} R_{u}(\widetilde{P})$ be a parabolic subgroup of $\widetilde{\Gamma}$ defined over $K$. Let $\widetilde{L}$ be a fixed Levi factor (defined over $K$ ) and let $R_{u}(\widetilde{P})$ be the unipotent radical of $\widetilde{P}$. Further, let $s \in \widetilde{P}(K), s=l u$, where $l \in \widetilde{L}$ and $u \in R_{u}(\widetilde{P})$. 
If $s \in \widetilde{\Gamma}(K)$, then $l \in \widetilde{L}(K)$ and $u \in R_{u}(\widetilde{P})(K)$. If, in addition, $s$ is a semisimple element, then $s$ is conjugate to $l$ in $\widetilde{P}$.

Proof. The first assertion follows from the uniqueness of the decomposition lu.

Further, if $s$ is semisimple, it is contained in a maximal torus in $\widetilde{P}$ which is contained in a Levi subgroup $L^{\prime}$. ([Sp, 8.4.4]). Since all Levi subgroups are conjugate in $\widetilde{P}([\mathbf{S p}, 16.1 .1])$ by elements of $\widetilde{P}$, one can find an element $p=l_{1} u_{1} \in \widetilde{P}$ where $l_{1} \in \widetilde{L}, u_{1} \in R_{u}(\widetilde{P})$ such that $p s p^{-1} \in \widetilde{L}$. Then $l_{1}^{-1} \mathrm{psp}^{-1} l_{1}=u_{1} s u_{1}^{-1}=l\left(l^{-1} u_{1} l\right) u u_{1}^{-1} \in \widetilde{L}$. Hence $\left(l^{-1} u_{1} l\right) u u_{1}^{-1}=1$ (because $\left.\left(l^{-1} u_{1} l\right) u u_{1}^{-1} \in R_{u}(\widetilde{P})\right)$ and therefore $l_{1}^{-1} p s p^{-1} l_{1}=l$.

Lemma 4.6. Let $s \in \widetilde{\Gamma}(K)$ be a semisimple element of $\widetilde{\Gamma}$ such that $C_{\widetilde{\Gamma}}(s)^{0}$ is not a torus. Then there exists a parabolic subgroup $\widetilde{P}$ of $\widetilde{\Gamma}$ defined over $K$ such that $s \in \widetilde{P}$.

Proof. The group $C_{\widetilde{\Gamma}}(s)^{0}$ is defined over $K$ ([Sp, 12.1.4]). Further, the condition $\operatorname{dim} K \leq 1$ implies that there exists a Borel subgroup $\widetilde{B}_{s}$ of $C_{\widetilde{G}}(s)^{0}$ which is also defined over $K([\mathbf{S t 1}, 10.2])$. Since $C_{\widetilde{\Gamma}}(s)^{0}$ is not a torus, the unipotent radical $R_{u}\left(\widetilde{B}_{s}\right)$ is not trivial. The group $\widetilde{U}_{1}=R_{u}\left(\widetilde{B}_{s}\right)$ is also defined over $K([\mathbf{S p}, 14.4 .5(\mathrm{v})])$. Further, let

$$
\begin{gathered}
\widetilde{N}_{1}=N_{\widetilde{G}}\left(\widetilde{U}_{1}\right), \widetilde{U}_{2}=\widetilde{U}_{1} \cdot R_{u}\left(\widetilde{N}_{1}\right), \widetilde{N}_{2}=N_{\widetilde{G}}\left(\widetilde{U}_{2}\right), \ldots, \\
\widetilde{U}_{i}=\widetilde{U}_{i-1} \cdot R_{u}\left(\widetilde{N}_{i-1}\right), \widetilde{N}_{i}=N_{\widetilde{G}}\left(\widetilde{U}_{i}\right), \ldots
\end{gathered}
$$

Then all members of (1) are closed subgroups of $\widetilde{\Gamma}$ and $\widetilde{U}_{k}=\widetilde{U}_{k+1}, \widetilde{N}_{k}=$ $\widetilde{N}_{k+1}$ for some positive integer $k([\mathbf{H u}, 30.3])$. Further, all groups in (1) are defined over $K$; indeed, the field $K$ is perfect and all groups are defined as normalizers of $K$-defined groups, their unipotent radicals, and the images of $K$-defined groups with respect to maps $\widetilde{U}_{i-1} \times R_{u}\left(\widetilde{N}_{i-1}\right) \longrightarrow \widetilde{U}_{i-1} \cdot R_{u}\left(\widetilde{N}_{i-1}\right)$, induced by multiplication in $\widetilde{G}$. Since $\widetilde{U}_{1}$ is connected, the last member $\widetilde{N}_{k}$ of this sequence is a parabolic subgroup of $\widetilde{\Gamma}([\mathbf{H u}, 30.3])$. From the definitions we have $s \in \widetilde{N}_{1} \leq \widetilde{N}_{k}$.

Now we can prove Theorem 1.3. Let $s \in G$ be a noncentral semisimple element. We may assume that $s$ is not a regular element of $\widetilde{G}$ (otherwise the statement follows from Theorem 1.1 and Property (d)). By Lemma 4.6 we have $s \in \widetilde{P}$ for some parabolic subgroup defined over $K$. Since $g \widetilde{P} g^{-1}=\widetilde{P}_{I}$ for some standard parabolic subgroup $\widetilde{P}_{I}$ and $g \in G$ ([Sp, 15.4.6]), we may assume $s \in \widetilde{P}_{I}$, where $I \subset \widetilde{\Pi}$ is a $\operatorname{Gal}(\bar{K} / K)$-invariant subset (note that the $\operatorname{group} \operatorname{Gal}(\bar{K} / K)$ acts on $\widetilde{\Pi}$ by permutation and the orbits of this action 
correspond to $\Pi$; see $[\mathbf{S t 1}$, Section 9$])$. Let $\widetilde{L}_{I}=\widetilde{T} \widetilde{G}_{I}$, where $\widetilde{G}_{I}=\left\langle X_{\alpha}\right.$ $\alpha \in\langle I\rangle\rangle$. Then $\widetilde{L}_{I}$ is a $K$-defined Levi factor of $\widetilde{P}_{I}$.

By Lemmas 4.5 and 4.2 we may assume $s \in \widetilde{L}_{I}$. (Indeed, by Lemma 4.5 we have en element $l \in \widetilde{L}_{I}(K)$ which is conjugate to $s$ in $\widetilde{P}_{I}$. By Lemma 4.2 the elements $s, l$ are conjugate by an element of the group $G$. Hence we may take the element $l \in C$ instead of $s$.)

Again by Lemma 4.6 we may assume that $C_{\widetilde{L}_{I}}(s)^{0}=\widetilde{T}^{\prime}$, where $\widetilde{T}^{\prime}$ is a maximal torus of $\widetilde{L}_{I}$ defined over $K$ (otherwise, we can take a smaller set $I$ using the same procedure as above). Note that the derived subgroup $\widetilde{L}_{I}$ is equal to $\widetilde{G}_{I}$ and therefore is a simply-connected semisimple group (because $\widetilde{G}$ is simply-connected). Hence $C_{\widetilde{L}_{I}}(s)^{0}=C_{\widetilde{L}_{I}}(s)([\mathbf{C 2}$, Theorem 3.5.6]) and thus

$$
C_{\widetilde{L}_{I}}(s)=\widetilde{T}^{\prime}
$$

Further, if $I=\emptyset$ we have $\widetilde{P}_{I}=\widetilde{B}$ and $\widetilde{T}^{\prime}=\widetilde{T}$. Hence $s \in \widetilde{T}(K)=T$. Since $s$ is a noncentral element of $G$, there exists a root $\alpha \in \Pi$ such that $s$ is not in the center of the group $T \widetilde{G}_{\alpha}(K)$ (here, $\widetilde{G}_{\alpha}=\left\langle X_{\beta} \mid \beta \in\left\langle I_{\alpha}\right\rangle\right\rangle$ where $I_{\alpha} \subset \widetilde{\Pi}$ is the $\operatorname{Gal}(\bar{K} / K)$-orbit of $\left.\alpha\right)$. Since the Borel subgroup $B_{\alpha}$ of $T \widetilde{G}_{\alpha}(K)$ (with respect to $T$ ) is not a normal subgroup, one can find an element $\gamma \in T \widetilde{G}_{\alpha}(K)$ such that $\gamma s \gamma^{-1}=\dot{w}_{\alpha} b$, where $w_{\alpha} \in W$ is the corresponding reflection and $b \in B_{\alpha}$. Hence $C \cap B \dot{w}_{\alpha} B \neq \emptyset$. Further, let $\omega \in W$. Then $\omega w_{\alpha} \omega^{-1}=w_{\beta}$, where $\beta=\omega(\alpha)$. Let $\dot{\omega}, \dot{w}_{\beta}$ be preimages of $\omega, w_{\beta}$ in the group $N$. Then $\dot{\omega} T \widetilde{G}_{\alpha}(K) \dot{\omega}^{-1}=T \widetilde{G}_{\beta}(K)$. The element $s^{\prime}=\dot{\omega} s \dot{\omega}^{-1}$ is not a central element in $T \widetilde{G}_{\beta}(K)$. Now, as above, we have $\gamma^{\prime} s^{\prime} \gamma^{\prime-1} \in B \dot{w}_{\beta} B$ for some $\gamma^{\prime} \in T \widetilde{G}_{\beta}(K)$. Thus, if $I=\emptyset$, the assertion of the theorem holds for $X=\{\alpha\}$.

Now we may assume that $I \neq \emptyset$ and Condition (2) holds.

We have $s=t g, t \in \widetilde{T} \cap C_{\widetilde{L}_{I}}\left(\widetilde{G}_{I}\right)$, and $g \in \widetilde{G}_{I}([\mathbf{H u}, 27.5])$. Note that the elements $t$ and $g$ do not necessarily belong to $G$ but $t, g \in \widetilde{L}_{I}\left(K^{\prime}\right)$ for some extension $K^{\prime} / K$. The element $s \in G$ is $\operatorname{Gal}(\bar{K} / K)$-invariant and $t \in Z\left(\widetilde{L}_{I}\right)$. Hence $g=h_{1} g_{1}$, where $h_{1} \in \widetilde{T}\left(K^{\prime}\right), g_{1} \in \widetilde{G}_{I}(K)$ (this follows from the Bruhat decomposition of $g$ ). Further, (2) implies that $g$ is a regular element of $\widetilde{G}_{I}$. If $\mathfrak{N}^{\prime}$ is a cross-section (defined over $K$ ) of regular semisimple conjugacy classes of $\widetilde{G}_{I}$ ([St1, Section 9]) then $h_{1} \mathfrak{N}^{\prime}$ is also a cross-section (defined over $K^{\prime}$ ) of regular semisimple conjugacy classes of $\widetilde{G}_{I}$. Hence the conjugacy class $C_{g}$ of $g$ in $\widetilde{G}_{I}$ intersects $h_{1} \mathfrak{N}^{\prime}$ in just one point. Thus the conjugacy class $C_{s}=t C_{g}$ of $s$ in $\widetilde{L}_{I}$ intersects $t h_{1} \mathfrak{N}^{\prime}$ also in one point $x$ (recall, $t \in Z\left(\widetilde{L}_{I}\right)$ ). Since the conjugacy class $C_{s}$ is defined over $K$ and the closed subset $t h_{1} \mathfrak{N}^{\prime}$ is also defined over $K$ (because $t h_{1}=s g_{1}^{-1} \in \widetilde{L}_{I}(K)$ ), 
the point $x$ is $\operatorname{Gal}(\bar{K} / K)$-invariant and therefore it belongs to $L_{I}(K)$. Since $s, x \in L_{I}(K) \leq G$ are conjugate in $L_{I}$ (and therefore in $\widetilde{G}$ ), we have $x=$ $\sigma s \sigma^{-1}$ for some $\sigma \in G$ (Lemma 4.2). Further,

$$
t h_{1} \mathfrak{N}^{\prime} \subset\left(\prod_{\alpha \in X} \dot{w}_{\alpha}\right) \widetilde{U},
$$

where $X \subset \Pi$ is the set of $\mathrm{Gal}(\bar{K} / K)$-orbits of $I \subset \widetilde{\Pi}$ and $w_{\alpha}$ in (3) is the product of basic reflections $w_{\gamma}$, where $\gamma$ runs through the orbit corresponding to $\alpha$ or $w_{\alpha}=w_{\gamma_{1}+\gamma_{2}}$ if such orbit consists of two roots $\gamma_{1}, \gamma_{2}$ such that $\gamma_{1}+\gamma_{2}$ is a root (see [St1, Section 9]). From (3) we obtain

$$
x=\sigma s \sigma^{-1} \in \widetilde{B} \prod_{\alpha \in X} \dot{w}_{\alpha} \widetilde{B} .
$$

Since $x \in G$, we have

$$
x=\sigma s \sigma^{-1} \in B \dot{w} B
$$

for some $w \in W$. But

$$
B \dot{w} B \subset \widetilde{B} \dot{w} \widetilde{B}
$$

From (4), (5), (6) we get

$$
w=\prod_{\alpha \in X} w_{\alpha}
$$

i.e., $w$ is a generalized $S$-Coxeter element of $W$. Now (5) and (7) imply that the conjugacy class of $s$ in $G$ intersects $B \dot{w} B$ for some generalized $S$-Coxeter element $w$ of $W$.

Suppose that $w^{\prime}=\omega w \omega^{-1}$ is also an $S$-Coxeter element of $W$ for some $\omega \in W$. Then $w^{\prime}=\prod_{\alpha \in Y} w_{\alpha}$ for some $Y \subset \Pi,|Y|=|X|$. Let $X^{\prime}=\{\omega(\alpha) \mid$ $\alpha \in X\}$. Then

$$
w^{\prime}=\prod_{\alpha \in Y} w_{\alpha}=\prod_{\beta \in X^{\prime}} w_{\beta} .
$$

The element $w^{\prime}$ is a Coxeter element of the root systems generated by $Y$ and $X^{\prime}$. It acts without fixed points on the vector space (over $\mathbb{R}$ ) generated by $Y$ and on the vector space generated by $X^{\prime}$. Moreover, $l\left(w^{\prime}\right)=|Y|=\left|X^{\prime}\right|$. Hence the vector spaces (over $\mathbb{R}$ ) generated by $Y$ and $X^{\prime}$ coincide (it is the $\left\langle w^{\prime}\right\rangle$-complement to the vector space of $w^{\prime}$-invariant vectors). Since $X$ is a simple root system for the root system $\langle X\rangle$, the set $X^{\prime}$ is a simple root system for $\left\langle X^{\prime}\right\rangle$. On the other hand, the set $Y$ is a simple root system for the root system $\langle Y\rangle$. Now $X^{\prime} \subset \omega(\Pi), Y \subset \Pi$ and the linear spaces generated by $X^{\prime}$ and $Y$ coincide. Moreover, the root subsystems $\left\langle X^{\prime}\right\rangle,\langle Y\rangle$ have the same Coxeter element $w^{\prime}$. Hence $\left\langle X^{\prime}\right\rangle=\langle Y\rangle$. Now let $I^{\prime}$ be a subset of $\widetilde{\Pi}$ 
that is $\operatorname{Gal}(\bar{K} / K)$-invariant and such that the set of Gal $(\bar{K} / K)$-orbits of $I^{\prime}$ coincides with $Y$. Since $\omega(\langle X\rangle)=\left\langle X^{\prime}\right\rangle=\langle Y\rangle$, we have

$$
\widetilde{G}_{I^{\prime}}=\left\langle X_{\beta} \mid \beta \in\left\langle I^{\prime}\right\rangle\right\rangle=\dot{\omega} \widetilde{G}_{I} \dot{\omega}^{-1} .
$$

From (8) we get

$$
\widetilde{L}_{I^{\prime}}=\widetilde{T} \widetilde{G}_{I^{\prime}}=\dot{\omega} \widetilde{L}_{I} \dot{\omega}^{-1} .
$$

Since $\omega \in W$, we can choose the preimage $\dot{\omega} \in G$. From (9)

$$
s^{\prime}=\dot{\omega} s \dot{\omega}^{-1} \in \widetilde{L}_{I^{\prime}} \cap G .
$$

Now we have a semisimple regular element $s^{\prime} \in \widetilde{L}_{I^{\prime}}(K)$. The same arguments as above show that there exists an element $\tau \in G$ such that $s^{\prime \prime}=\tau s^{\prime} \tau^{-1} \in$ $B \dot{w}^{\prime \prime} B$, where

$$
w^{\prime \prime}=\prod_{\beta \in Y} w_{\beta}
$$

(the order of the roots $\beta$ in this product can be different from the order of the roots $\alpha$ in the product corresponding to $\left.w^{\prime}\right)$. By Lemma 2.4 there exists an $S$-ascent from $w^{\prime \prime}$ to $w^{\prime}$ (both elements are $Y$-Coxeter elements for the Weyl group of the system $\langle Y\rangle)$. Proposition 3.4 implies

$$
\delta s^{\prime \prime} \delta^{-1} \in B \dot{w}^{\prime} B
$$

for some $\delta \in G$.

The inclusions (5) and (10) show that the conjugacy class $C$ of $s$ in $G$ intersects all Bruhat cells $B \dot{w}^{\prime \prime \prime} B$, where $w^{\prime \prime \prime}$ runs through all generalized $S$-Coxeter elements that are conjugate to $w$. Now let $\widetilde{w} \in W$ be an element from the conjugacy class of $w$. Proposition 3.3 implies that there exists an $S$-ascent from some generalized $S$-Coxeter element $w^{\prime \prime \prime}$ to $\widetilde{w}$. Now the assertion of the theorem follows from Proposition 3.4.

Theorem 1.3 has been proved.

\section{Remarks to Theorem 1.3.}

1. Intersection with a parabolic subgroup. In the proof of Theorem 1.3 we showed that

$$
C \cap P_{X} \neq \emptyset
$$

for every noncentral semisimple conjugacy class $C$ that is not regular, where $X \varsubsetneqq \Pi$ and $P_{X}=B W_{X} B$ is the corresponding parabolic subgroup (if $K$ is a perfect field and $\operatorname{dim} K \leq 1)$. More generally, Equation (**) holds for every noncentral conjugacy class $C$ that is not a regular semisimple class (if $K$ is a perfect field and $\operatorname{dim} K \leq 1$ ). Indeed, we consider the Jordan decomposition $g=s u$ of an element $g \in C$. Applying the same construction as in Lemma 4.6, we get a parabolic subgroup $P$ which is defined over $K$ and contains $s, u$. Then by an appropriate conjugation we can embed $g$ in 
a standard parabolic subgroup. (Note, if $K$ is a finite field, then Condition $(* *)$ is a consequence of the properties of the Steinberg representation $[\mathbf{C 2}$, Proposition 6.4.5].)

2. The condition: $\operatorname{dim} K \leq \mathbf{1}$. The example below shows that if this condition does not hold, the conclusion of Theorem 1.3 may be false.

Let $n=4 k$ and let $V$ be a linear space over the real number field $\mathbb{R}$ such that $\operatorname{dim} V=4 k$. Further, let $\left\{e_{1}, \ldots, e_{4 k}\right\}$ be a fixed basis of $V$ and let $V^{+}=\left\langle e_{1}, \ldots, e_{2 k}\right\rangle, V^{-}=\left\langle e_{2 k+1}, \ldots, e_{4 k}\right\rangle$. Further, let $\left(x_{1}, \ldots, x_{4 k}\right)$ be the coordinates of an element in $V$ with respect to the basis $\left\{e_{i}\right\}$ and let $\Phi=$ $x_{1}^{2}+\cdots+x_{2 k}^{2}-x_{2 k+1}^{2}-\cdots-x_{4 k}^{2}$. Let $\Omega=\Omega(V, \Phi)=[S O(V, \Phi), S O(V, \Phi)]$. Then $\Omega$ is a Chevalley group in the sense of [St2], corresponding to the root system $D_{2 k}$. Let $g \in G L(V)$ be the linear operator such that $\left.g\right|_{V^{+}}=$ $-1,\left.g\right|_{V^{-}}=1$. One can easily check that $g \in \Omega$ and $g u g^{-1} \neq u^{ \pm 1}$ for every nontrivial unipotent element $u \in \Omega$ (the latter follows from the fact that $v \pm g(v)$ is not an isotropic vector if $v \neq 0$ is isotropic). Hence the element $g$ cannot normalize any nontrivial unipotent subgroup of $\Omega$ and therefore $g$ cannot belong to any proper parabolic subgroup of $\Omega$. This implies that a preimage $\hat{g}$ of $g$ in $G=\operatorname{Spin}_{4 k}(\mathbb{R})$ (with respect to the natural homomorphism $G \longrightarrow \Omega$ ) also cannot belong to a proper parabolic subgroup of $G$. Hence $C \cap B w_{X} B=\emptyset$ for every $X \subset \Pi$, where $C$ is the conjugacy class of $\hat{g}$ in $G, B$ is a Borel subgroup of $G$, and $\Pi$ is a simple root system corresponding to $\widetilde{G}=\mathbf{S p i n}_{4 k}$ (note, $B W_{X} B=P_{X}$ is a standard parabolic subgroup).

3. The ordered set of $\mathfrak{X}_{\mathbf{C}}$. Recall, for any set $X \subset \Pi$ we define $w_{X}=$ $\prod_{\alpha \in X} w_{\alpha}$, where the product can be taken in any fixed order. For the set

$$
\mathfrak{X}_{C}=\left\{X \subset \Pi \mid C \cap B \dot{w}_{X} B \neq \emptyset\right\}
$$

one can consider the natural order with respect to inclusion.

Let $G=S L_{n}(\mathbb{C})$ and $C$ a noncentral semisimple conjugacy class. Let $\lambda(C)=\left(\lambda_{1}, \ldots, \lambda_{r}\right)$ be the partition of $n$, i.e., $\lambda_{1} \geq \cdots \geq \lambda_{r}$, where $\lambda_{1}+\cdots+$ $\lambda_{r}=n$, which corresponds to the multiplicities of eigenvalues of elements of $C$ (i.e., $\lambda_{1}$ is the biggest multiplicity, then $\lambda_{2}$, etc.) and let $\lambda^{*}(C)$ be the dual partition (i.e., the rows and columns of $\lambda$ are interchanged). Further, to every partition $\mu=\left(\mu_{1}, \ldots, \mu_{s}\right)$ of $n$ we assign a subset $X(\mu) \subset \Pi=$ $\left\{\alpha_{1}, \ldots, \alpha_{n-1}\right\}$, namely,

$$
X(\mu) \stackrel{\text { def }}{=} \Pi \backslash\left\{\alpha_{\mu_{1}}, \alpha_{\mu_{1}+\mu_{2}}, \ldots, \alpha_{\mu_{1}+\cdots+\mu_{s-1}}\right\} .
$$

It is easy to see that $X\left(\lambda^{*}(C)\right)$ is a maximal element of $\mathfrak{X}_{C}$. Moreover, every maximal element $Y \in \mathfrak{X}_{C}$ is $W$-conjugate to $X\left(\lambda^{*}(C)\right)$. Thus we have just one conjugacy class $\left\{w w_{X} w^{-1}\right\}$ in $W$ for each maximal $X \in \mathfrak{X}_{C}$.

For other types of groups we can have several conjugacy classes in $W$ of elements of the form $w_{X}$, where $X \in \mathfrak{X}_{C}$ is a maximal element. Say, consider 
the root system $R=B_{2}=\left\langle\alpha_{1}, \alpha_{2}\right\rangle$, where $\alpha_{1}=\varepsilon_{1}-\varepsilon_{2}$ and $\alpha_{2}=\varepsilon_{2}$ (in the notation of [Bou] $)$, and let $G$ be the corresponding simple and simply connected group over $\mathbb{C}$. Let $g=h_{\varepsilon_{1}}(t) h_{\varepsilon_{2}}\left(t^{-1}\right) \in G$ be a semisimple element, where $h_{\varepsilon_{1}}(t), h_{\varepsilon_{2}}\left(t^{-1}\right)$ are the corresponding root semisimple elements (in the notation of [St2]) and $t \neq \pm 1$. Let $C$ be the conjugacy class of $g$. Then $\left\{\alpha_{1}\right\}$ and $\left\{\alpha_{2}\right\}$ both are maximal elements of $\mathfrak{X}_{C}$. Thus here we have two different conjugacy classes in $W$ of elements $w_{X}$ for maximal $X$ in $\mathfrak{X}_{C}$.

\section{Appendix.}

The following result as well as the line of proof was pointed out to the second author by T.A. Springer in the discussion of relevant questions:

Proposition 5.1. Let $\widetilde{G}$ be a simple algebraic group defined over an algebraically closed field $\bar{K}$ and let $G=\widetilde{G}(\bar{K})$. Further, let $C$ be the conjugacy class of a regular element of $G$. Then $C \cap B \dot{w} B \neq \emptyset$ for every $w \in W$.

Proof. For $b \in B$ we put

$$
\mathfrak{O}_{B}(b)=\left\{x b x^{-1} \mid x \in B\right\} .
$$

Lemma 5.2. There exists a nonempty finite set $\left\{b_{1}, \ldots, b_{n}\right\} \subset C \cap B$ such that

$$
C \cap B=\bigcup_{1 \leq i \leq n} \mathfrak{O}_{B}\left(b_{i}\right)
$$

Proof. Let $x=s_{1} u_{1}, y=s_{2} u_{2} \in B$ be two regular elements, where $s_{1}, s_{2} \in T$ and $u_{1}, u_{2} \in U$. We show

$$
\mathfrak{O}_{B}(x)=\mathfrak{O}_{B}(y) \text { if and only if } s_{1}=s_{2} .
$$

Indeed, "only if" is obvious. Now let

$$
b_{1}=s u_{1}, b_{2}=s u_{2}, s \in T, u_{1}, u_{2} \in U .
$$

Since we can consider the Jordan decompositions of $x, y$ as elements of $B$, we may assume that (12) gives the Jordan decompositions of $b_{1}$ and $b_{2}$. Put $\Gamma=\left[C_{G}(s), C_{G}(s)\right], B_{\Gamma}=B \cap \Gamma$. Then (12) implies $u_{1}, u_{2} \in B_{\Gamma}$. Moreover, the elements $u_{1}, u_{2}$ are regular unipotent elements of $\Gamma([\mathbf{S t 1}, 3.7])$ and therefore the elements $u_{1}, u_{2}$ are conjugate in $B_{\Gamma}$ (see $[\mathbf{C 2}$, the proof of Proposition 5.1.3]). Hence we have (11).

Now let $b=s u \in B, s \in T, u \in U, g \in G, g b g^{-1} \in B$. Further, let $g \in B \dot{w} B$. Then $g b g^{-1}=w(s) u^{\prime}$ for some $u^{\prime} \in U$. Together with (11), this implies our assertion.

Lemma 5.3. Let $b \in C \cap B$ be a fixed element and let $w \in W$. Then every irreducible component $\mathfrak{C}_{w}$ of $\bar{C} \cap \overline{B \dot{w} B}$ such that $\mathfrak{O}_{B}(b) \subset \mathfrak{C}_{w}$ satisfies the following condition:

$$
\operatorname{dim} \mathfrak{C}_{w}=\operatorname{dim} \bar{C}+\operatorname{dim} \overline{B \dot{w} B}-\operatorname{dim} G .
$$


Proof. Since $b$ is a regular element, $\operatorname{dim} C_{B}(b)=\operatorname{rank} G([\mathbf{S t 1}, 3.11])$. If $\mathfrak{C}_{1}$ is an irreducible component of $\bar{C} \cap B$ containing $\mathfrak{O}_{B}(b)$, then Lemma 5.2 implies $\mathfrak{C}_{1}=\overline{\mathfrak{O}_{B}(b)}$ and, therefore,

$$
\operatorname{dim} \mathfrak{C}_{1}=\operatorname{dim} B-\operatorname{rank} G=\operatorname{dim} \bar{C}+\operatorname{dim} B-\operatorname{dim} G .
$$

Let $\mathfrak{O}_{B}(b) \subset \mathfrak{C}_{w}$ for some irreducible component $\mathfrak{C}_{w}$ of $\bar{C} \cap \overline{B \dot{w} B}$. Suppose

$$
\operatorname{dim} \mathfrak{C}_{w}>\operatorname{dim} \bar{C}+\operatorname{dim} \overline{B \dot{w} B}-\operatorname{dim} G .
$$

Since $B$ is a closed subset of $\overline{B \dot{w} B}$ ([Sp, 8.15]) and $\mathfrak{C}_{1}$ is an irreducible component of $\mathfrak{C}_{w} \cap B$, we have

$$
\operatorname{dim} \mathfrak{C}_{1} \geq \operatorname{dim} \mathfrak{C}_{w}+\operatorname{dim} B-\operatorname{dim} \overline{B \dot{w} B} .
$$

Now (14) and (15) contradict (13). Thus we have our statement.

Now we return to the proof of Proposition 5.1.

Take $\mathfrak{C}_{w}$ as in Lemma 5.3. Assume $C \cap B \dot{w} B=\emptyset$. Then

$$
\mathfrak{C}_{w} \subset \bigcup_{w^{\prime}<w} B \dot{w}^{\prime} B=\bigcup_{w^{\prime}<w} \overline{B \dot{w}^{\prime} B}
$$

([Sp, 8.15]). From (16) we have $\mathfrak{C}_{w} \subset \overline{B \dot{w}^{\prime} B}$ for some $w^{\prime}<w$ and we may consider $\mathfrak{C}_{w}$ as an irreducible component of $\bar{C} \cap \overline{B \dot{w}^{\prime} B}$ that contains $\mathfrak{O}_{B}(b)$. Then, by Lemma 5.3, we have

$$
\operatorname{dim} \mathfrak{C}_{w}=\operatorname{dim} \bar{C}+\operatorname{dim} \overline{B \dot{w}^{\prime} B}-\operatorname{dim} G .
$$

But (17) contradicts Lemma 5.3 because $\operatorname{dim} \overline{B \dot{w}^{\prime} B}<\operatorname{dim} \overline{B \dot{w} B}$. This proves Proposition 5.1.

\section{References}

[Bo] A. Borel, Linear Algebraic Groups, 2nd ed., Graduate Texts in Mathematics, 126, Springer-Verlag, New York, 1991, MR 1102012, Zbl 0726.20030.

[Bou] N. Bourbaki, Éléments de Mathématique. Groupes et Algèbre de Lie, Chap. IV, V, VI, 2ème édition, Masson, Paris, 1981, MR 1890629, Zbl 0483.22001.

[C1] R.W. Carter, Simple Groups of Lie Type, Reprint of the 1972 original, Wiley Classics Library, John Wiley and Sons, New York, 1989, MR 1013112, Zbl 0723.20006.

[C2] - Finite Groups of Lie Type. Conjugacy Classes and Complex Characters, Reprint of the 1985 original, Wiley Classics Library, John Wiley and Sons, Chichester, 1993, MR 1266626, Zbl 0567.20023.

[EG] E.W. Ellers and N. Gordeev, Intersection of conjugacy classes of Chevalley groups with Gauss cells, J. Algebra, 220 (1999), 591-611, MR 1717361, Zbl 942.20030.

[EGH] E.W. Ellers, N. Gordeev and M. Herzog, Covering numbers for Chevalley groups, Israel J. Math., 111 (1999), 339-372, MR 1710745, Zbl 941.20050. 
[GP] M. Geck and G. Pfeiffer, Characters of Finite Coxeter Groups and Iwahori-Hecke Algebras, London Mathematical Society Monographs, New Series, 21, The Clarendon Press, Oxford University Press, New York, 2000, MR 1778802, Zbl 0996.20004.

[GS] N. Gordeev and J. Saxl, Products of conjugacy classes in Chevalley groups I. Extended covering numbers, Israel J. Math., 130 (2002), 207-248, MR 1919378, Zbl 1007.20041.

[Hu] J.E. Humphreys, Linear Algebraic Groups, Graduate Texts in Mathematics, 21, Springer-Verlag, New York-Heidelberg-Berlin, 1975, MR 0396773, Zbl 0325.20039.

[K] N. Kawanaka, Unipotent elements and characters of finite Chevalley groups, Osaka J. Math., 12(2) (1975), 523-554, MR 0384914, Zbl 0314.20031.

[Sp] T.A. Springer, Linear Algebraic Groups, 2nd edition, Progress in Mathematics, 9, Birkhäuser Boston, Boston MA, 1998, MR 1642713, Zbl 0927.20024.

[St1] R. Steinberg, Regular elements of semisimple algebraic groups, Inst. Hautes Études Sci. Publ. Math., 25 (1965), 49-80, MR 0180554, Zbl 0136.30002.

[St2] Lectures on Chevalley Groups, Notes prepared by John Faulkner and Robert Wilson, Yale Univ., New Haven CT, 1968, MR 0466335, Zbl 0307.22001.

[V] N.A. Vavilov, Bruhat decomposition of two-dimensional transformations (Russian), Vestnik Leningrad. Univ. Mat. Mekh. Astronom., 3 (1989), 3-7; translation in Vestnik Leningrad Univ. Math., 22(3) (1989), 1-6, MR 1055331, Zbl 0716.20024.

[VS] N.A. Vavilov and A.A. Semenov, Bruhat decomposition for long root tori in Chevalley groups (Russian), Zap. Nauchn. Sem. Leningrad. Otdel. Mat. Inst. Steklov. (LOMI), 175, 1989, Koltsa i Moduli, 3, 12-23, 162; translation in J. Soviet Math., 57(6) (1991), 3453-3458, MR 1047233, Zbl 0746.20024.

Received August 12, 2002 and revised June 16, 2003. Research was supported by NSERC Canada Grant A7251 and by INTAS-99-00817.

Department of Mathematics

UNIVERSITY OF TORONTO

100 St.George Street, Toronto

ONTARIO M5S 3G3

CANADA

E-mail address: ellers@math.toronto.edu

Russian State Pedagogical University

MOIJKA 48

St.Petersburg 191-186

Russia

E-mail address: nickgordeev@mail.ru 